\title{
Effect of High Flux Versus Low Flux Hemodialysis on Some Cardiovascular Risk Factors in Egyptian Children with End Stage Renal Disease.
}

\author{
Ahlam Badawy', Dalia A. Nigm², Hekma Saad Farghaly', Yasser Gamal ${ }^{3}$ \\ 1. Pediatric Department, Faculty of Medicine, Assiut University, Egypt. \\ 2. Clinical Pathology Department, Faculty of Medicine, Assiut University, Egypt. \\ 3. Pathology Department, Faculty of Medicine, Assiut University, Egypt.
}

\section{Abstract}

Background Cardiovascular disease remains as the main cause of mortality in children with end stage renal disease (ESRD).Among the cardiovascular risk factors, dyslipidemia and inflammation are of great importance as they are both prevalent and modifiable risk factors. We aim to evaluate the effect of permeability of low-flux versus high-flux dialyzer on such 2 factors in children on regular hemodialysis (HD).

\section{Methods}

48 children with ESRD were randomized into 2 groups. Group A used low-flux dialyzer, group B used high-flux dialyzer for 6 months. An initial and 6th month blood samples for lipid profile, serum albumin and serum high sensitivity CRP (hs CRP) were obtained. Statistical analysis was done.

\section{Results}

In the high flux group, there was a statistically significant difference in total cholesterol, HDL-cholesterol, triglyceride and hs CRP levels after 6 months when compared to baseline values of the same group, while LDL cholesterol and serum albumin showed no significant change. In the low flux group, results after 6 months didn't show significant change in previously mentioned parameters when compared with its baseline values of the same group. Comparing these parameters between the 2 groups at 6 months, shows that high flux group has significantly lower total cholesterol, triglyceride, and hs CRP levels.

\section{Conclusion}

This study has demonstrated for the first time improvement of some markers of dyslipidemia and inflammation by using high-flux HD in children with ESRD. These results suggest that high-flux dialysis may benefit such young suffering population by reducing the atherogenic risk, cardiovascular morbidity and mortality.

\section{Keywords}

Dyslipidemia, hemodialysis, high flux, uremic children.

\section{Correspondence}

Ahlam Badawy

Department of Pediatrics, Faculty of Medicine, Assiut University, Assiut, Egypt .

E-mail: dr.ahlam_ali@yahoo.com

\author{
geget : The Journal of the Egyptian Society of Pediatric Nephrology and Transplantation (ESPNT) \\ geget https://geget.journals.ekb.eg/ \\ Published by ESPNT http://espnt.net/ \\ Cohosted by Egyptian Knowledge Bank https://www.ekb.eg
}




\section{Introduction}

Cardiovascular disease (CVD) is considered the primary cause of death in adults with end stage renal disease (ESRD), as it is responsible for nearly $50 \%$ of deaths. The data are more alarming for children, as CVDspecific death rates in children and young adults with ESRD have increased over the last two decades. They are approximately 100 times more than normal comparably aged populations [1].

Chronic kidney disease (CKD) carries constellation of risk factors for CVD. Such risk factors include traditional factors as (hypertension, dyslipidemia, diabetes) and nontraditional one as (anemia, inflammation, mineral metabolism disorders, oxidative stress) [2].To reduce the risk of CVD related mortality in dialysis patients, an integrated approach is required which aims to control dyslipidemia, malnutrition, inflammation and other relevant risk factors.

Among the important cardiovascular risk factors, dyslipidemia is an important focus of clinical research because, it is both a highly prevalent (it is found in 39$65 \%$ of patients) and a potentially modifiable risk factor [3]. Effect of high flux hemodialysis on uremia-related dyslipidemia is uncertain, and results are conflicting [3].

It is now well-documented that ESRD is associated with a state of chronic inflammation [4]. Circulating inflammatory cytokines represent an important link between CKD and CVD e.g. elevated circulating levels of IL 6 have been linked to hypertension, left ventricular hypertrophy $(\mathrm{LVH})$, and atherosclerosis among patients with ESRD. In addition serum CRP is found to be a longterm predictor of CVD related mortality in patients with CKD [5].

As children represent a population without preexisting symptomatic CVD, children with CKD may receive significant benefit from attempts to prevent and treat uremia related risk factors for CVD. So here we addressed the question whether a high-flux dialysis has an impact on 2 major modifiable risk factors for CVD which are dyslipidemia and inflammatory process in children on regular hemodialysis (HD). This study approaches the issue through investigating the effect of low-flux versus highflux dialysis membranes on some parameters of lipid metabolism, serum albumin and serum hs CRP ( as a sensitive marker of inflammation).

\section{Patients and Method}

Study design and participants Forty eight children with ESRD, who were on regular HD in Pediatric Nephrolgy and Dialysis Unit at tertiary care center of Children University Hospital, were included in this prospective study. At time of start of the study, whole number of patients attending the unit for regular hemodialysis were 75 . However, only 48 patients had fulfilled inclusion criteria and were eligible for enrollment in this study. Inclusion criteria were as follow: age less than 18 years old, had ESRD with regular hemodialysis for at least 6 months, dry weight was relatively stable, and arteriovenous fistula was done for performance of hemodialysis. Only patients who were free of any infection (according to clinical and laboratory evaluation) for at least 4 weeks before enrollment in the study were included. Cases with history of glomerulonephritis as an etiology of renal failure were excluded from the study as it may be associated with abnormal inflammatory milieu of the patient.

Hemodialysis was done 3 times weekly; 4 hours per session under standard heparin doses with Fresenius 4008B machines using single use polysulfone filters, pediatric lines and containing a diasafe device for water pureness. The study was approved by the local Institutional Review Board of the Faculty of Medicine. An informed written consent, in accordance with the Ethical Committee guidelines, was taken from guardians of all cases and controls.

Etiologies for renal failure included polycystic kidney disease, renal hypoplasia and dysplasia, nephrocalcinosis, refluxing nephropathy, and nephronophsiathesis. Twenty healthy children of comparable age and sex (with no history of any renal disease and with normal urine analysis and renal function) were enrolled in the study as a control group after their informed consent.

Upon enrollment into the study, low flux dialysis was used for 6 weeks for all patients using a polysulfone dialyzer (F3, 4 HPS; Fresenius Medical Care, Bad Homburg, Germany) with a surface area of $0.4,0.7 \mathrm{~m}^{2}$ according to the patient weight and surface area. Thereafter, patients received serial numbers according to their first registration in the unit. Patients with odd numbers were allocated to group A, and patients with even numbers were allocated to group B with 24 patients in each. Group A continued HD treatment using a low-flux polysulfone dialyzer, while patients of group B started treatment with polysulfone high-flux dialyzer (FX 40 Helixone; Fresenius Medical Care, Bad Homburg, Germany) for 6 months duration. Randomized groups were blinded to team of laboratory and statistical data. Standards of ultra-purification of water and dialysate were met during process of hemodialysis. During the 6 months, 8 patients failed to complete the study due to occurrence of infection ( 3 patients), failure of follow up ( 2 patients) or death of the patient ( 3 patients), where 3 of them dropped from group A and 5 from group B. An initial and 6th month blood samples were drawn from the non arteriovenous fistula arm before start of the mid-week dialysis session. Careful history and examination were done including assessment of weight, height, calculation of body mass index (BMI), and $\mathrm{z}$ score for BMI. Blood pressure was reported as the average of two measurements. Antihypertensive medications were recorded. Hypertension was present if either systolic or diastolic blood pressure exceeded the 95th centile using age-sex-height-specific blood pressure percentiles, or if the patients were receiving antihypertensive treatment. None of the patients of both groups had received antibiotic, anti-inflammatory, or any drug that affect lipid metabolism as statins, thiazides, B blockers, or steroid throughout period of the study. Patients with diabetes, uncontrolled hypertension, and patients who received any drug that affect lipid metabolism or inflammatory process were excluded from the study. Both groups have received phosphate binders according to the same protocol. 
Sampling and processing Blood samples were collected after overnight $12 \mathrm{~h}$ fasting. Five mL of blood was obtained from each subject and was divided into EDTA tube $(2.0 \mathrm{~mL})$ and plain tube $(3.0 \mathrm{~mL})$. Serum samples were stored at $-20^{\circ} \mathrm{C}$ until the time of performing the analysis.

For all patients and control, the following investigations were done:

*Complete blood count (CBC): On CELL-DYN 3700

* Kidney function test: On Siemens Dimension RL Max

*Liver function: On Siemens Dimension RL Max

*Lipid profile: On Siemens Dimension RL Max, total cholesterol and triglyceride concentrations were estimated using enzymatic methods (CHOD-PAP and GPO-PAP, respectively; Roche Diagnostics, Mannheim, Germany). HDL cholesterol was determined after precipitation with phosphotungstic acid / magnesium chloride. LDL cholesterol was measured directly with a commercially available direct LDL-Cassay (LDL-C Plus assay; Roche Diagnostics).

*hs CRP: On BN Prospec

Dyslipidemia was defined as total cholesterol, LDL cholesterol or triglyceride $>95$ th centile, and/or HDL cholesterol $<5$ th centile for age and sex [6] and/or a nonHDL cholesterol (total cholesterol - HDL cholesterol) $>145 \mathrm{mg} / \mathrm{dl}$ [7].

Statistical analysis The data were tested for normality using the Anderson-Darling test and for homogeneity variances prior to further statistical analysis. Categorical variables were described by number and percent $(\mathrm{N}, \%)$, where continuous variables described by mean and standard deviation (Mean, SD). Median was taken for skewed continuous variables. Chi-square and fisher exact tests used to compare between categorical variables where compare between continuous variables by paired and unpaired t-test. A two-tailed $\mathrm{p}<0.05$ was considered statistically significant. All analyses were performed with the IBM SPSS 20.0 software.

\section{Results}

Characteristics of the study population including 48 patients (with ESRD and on regular hemodialysis) compared to 20 control are shown in Table 1. There was no significant difference in demographic data between patients and control with slight female predominance in both. As regard biochemical finding, blood levels of total cholesterol, triglyceride, and hs CRP were significantly higher in patients compared with control. Serum LDL was higher in patients than controls but with no significant difference ( $p$ value 0.603). As regard HDL, it was significantly lower in patients than control group. Data are demonstrated in (Table 1)

Table 2 show that dyslipidemia was observed in $81.3 \%$ of uremic children. Elevated levels of triglyceride, total cholesterol, and LDL were present in $60.4 \%, 10.4 \%$, and $6.3 \%$ of patients respectively; while $54.1 \%$ patients had low serum HDL cholesterol. Non-HDL cholesterol (total cholesterol - HDL cholesterol) was $>145 \mathrm{mg} / \mathrm{dl}$ in $20.8 \%$ of patients. (Table 2).

Base line demographic, and clinical data of A and B sub groups of patients did not differ significantly between both subgroups as depicted in table 3 . There was no significant difference between both subgroups in age, sex, or number of patients with $\mathrm{z}$ score ( BMI) below 3 SD. Mean dialysis vintage showed insignificant statistical difference. Also there was no difference in number of patients who had hypertension. Biochemical parameters, such as total protein, albumin, WBCs, $\mathrm{Hb}$, Platelets were not significantly different between the two groups of patients as shown in Table (3).

Mean ultrafiltration rate throughout the period of the study was $593.0 \pm 90.72 \mathrm{ml} / \mathrm{h}$ in low flux group versus $621.14 \pm 108.3 \mathrm{ml} / \mathrm{h}$ in high flux group with $\mathrm{P}$ value 0.275 . Mean values of blood flow rate and dialysate flow rate didn't show a significant difference throughout the study period. 
Table 1: Demographic, and biochemical parameters in patients with ESRD and controls.

\begin{tabular}{|c|c|c|c|}
\hline & Case: N (48) & Control: N (20) & P value \\
\hline Age & $13.08 \pm 3.008$ & $13.86 \pm 2.89$ & 0.385 \\
\hline $\begin{array}{c}\text { Sex (No., \%) } \\
\text { Memale }\end{array}$ & $21(43.75 \%)$ & $11(55 \%)$ & 0.535 \\
\hline Cholesterol(mg/dl) & $27(56.25 \%)$ & $131.81 \pm 18.63$ & 0.003 \\
\hline Triglycerides(mg/dl) & $160.52 \pm 38.95$ & $106 \pm 15.95$ & 0.001 \\
\hline HDL (mg/dl) & $168.1 \pm 78.86(152.3) *$ & $39.60 \pm 2.64$ & 0.003 \\
\hline LDL (mg/dl) & $29.50 \pm 8.62$ & $85.6+35.70(77.5) *$ & 0.603 \\
\hline hs CRP (mg/l) & $92.41 \pm 36.81(88.4) *$ & $1.35 \pm 0.33$ & 0.000 \\
\hline Calcium (mg/dl) & $10.16 \pm 6.82(8.71) *$ & $9.46 \pm 0.71$ & 0.001 \\
\hline Phosphorus (mg/dl) & $8.08 \pm 2.11$ & $4.14 \pm 0.51$ & 0.000 \\
\hline Total protein (g/l) & $7.01 \pm 2.80$ & $74.15 \pm 5.12$ & 0.013 \\
\hline Hb (g/dl) & $69.09 \pm 8.05$ & $13.37 \pm 0.57$ & 0.000 \\
\hline
\end{tabular}

Table 2: Patterns of dyslipidemia in studied children with ESRD.

\begin{tabular}{|c|c|c|}
\hline Lipid Profile & No & \% \\
\hline Increased Triglyceride levels & 29 & 50.4 \\
\hline Decreased HDL cholesterol levels & 26 & 6.3 \\
\hline Increased LDL cholesterol levels & 3 & 20.8 \\
\hline Decreased Non-HDL cholesterol & 10 & 81.3 \\
\hline Total & 39 & \\
\hline
\end{tabular}

HDL, high-density lipoprotein; LDL, low-density lipoprotein.

After 6 months, there were 21 patients in low flux group, and 19 patients in high flux group. Percentage of patients who had hypertension and those with $\mathrm{Z}$ score (BMI) below 3 SD did not differ significantly from their percentage at the start of the study in both subgroups. As regard markers of inflammations and lipid profile: Absolute values were compared within the same group over time. In the high flux group, there was a statistically significant difference noted in total cholesterol, HDL-cholesterol, triglyceride and hs CRP levels after 6 months when compared to baseline values in the same group, while LDL cholesterol and serum albumin showed no significant change. In the low flux group, results after 6 months didn't show significant change when compared with its baseline values in the same group. In addition, mean values of lipid profile, serum albumin, and hs CRP were compared between both subgroups. Data are shown in Table 4. Other laboratory parameters (urea, creatinine, calcium $\mathrm{Hb}, \mathrm{WBCs}$, platelet) show no significant change over 6 months duration in both subgroups. Serum phosphorus shows significant improvement in high flux group. Percentages of change of values of lipid profile, and h CRP between time 0 and 6 months were calculated and shown in Graph (1). 
Table 3: Baseline demographic, clinical, and laboratory findings in the 2 subgroups of low and high flux hemodialysis.

\begin{tabular}{|c|c|c|c|}
\hline Item & Low flux “n=24" & High flux "n=24" & P-value \\
\hline $\begin{array}{c}\text { Age "years" } \\
\text { Mean } \pm \text { SD }\end{array}$ & $12.81 \pm 3.22$ & $13.62 \pm 2.53$ & 0.483 \\
\hline $\begin{array}{c}\text { Sex: } \mathbf{n .}(\boldsymbol{\%}) \text { Male } \\
\text { Female }\end{array}$ & $\begin{array}{c}8(33.3 \%) \\
16(66.7 \%) \\
\end{array}$ & $\begin{array}{l}11(45.8 \%) \\
13(54.2 \%) \\
\end{array}$ & 0.907 \\
\hline 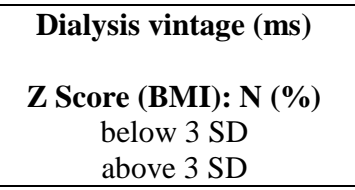 & $\begin{array}{l}18.81 \pm 10.26 \\
10(41.67 \%) \\
14(58.33 \%) \\
\end{array}$ & $\begin{array}{l}19.23 \pm 10.87 \\
11(45.83 \%) \\
13(54.16 \%) \\
\end{array}$ & 0.486 \\
\hline $\begin{array}{c}\text { Hypertension: } \mathbf{N}(\%) \\
\text { Yes } \\
\text { No }\end{array}$ & $\begin{array}{l}18(75.0 \%) \\
6(25.0 \%)\end{array}$ & $\begin{array}{c}20(83.33 \%) \\
4(16.67 \%)\end{array}$ & 0.275 \\
\hline $\begin{array}{c}\text { Total cholesterol }(\mathbf{m g} / \mathbf{d l}) \\
\text { TG(mg/dl) } \\
\text { HDL }(\mathbf{m g} / \mathbf{d l}) \\
\text { LDL }(\mathbf{m g} / \mathbf{d l}) \\
\text { hCRP(mg/L) } \\
\text { Urea }(\mathbf{u m o l} / \mathbf{l}) \\
\text { Creatinine(umol/l) } \\
\text { Calcium(mg/dl) } \\
\text { Phosphorus }(\mathbf{m g} / \mathbf{d l}) \\
\end{array}$ & $\begin{array}{c}158.81 \pm 33.99 \\
171.67 \pm 74.61(145.6) * \\
29.67 \pm 9.12 \\
97.00 \pm 45.86(72.5) * \\
11.81 \pm 5.92(8.71) * \\
34.62 \pm 10.14 \\
744.81 \pm 220.4 \\
8.58 \pm 1.87 \\
6.99 \pm 2.08\end{array}$ & $\begin{array}{c}164.08 \pm 49.05 \\
181.54 \pm 88.68(172.56) * \\
29.15 \pm 7.81 \\
87.42 \pm 23.59 \\
14.46 \pm 5.56(12.9) * \\
34.11 \pm 10.82 \\
894 \pm 261.66 \\
7.94 \pm 2.4 \\
7.2 \pm 2.52\end{array}$ & $\begin{array}{l}0.694 \\
0.463 \\
0.863 \\
0.078 \\
0.154 \\
0.894 \\
0.131 \\
0.700 \\
0.528 \\
\end{array}$ \\
\hline $\begin{array}{c}\text { Total protein }(\mathrm{g} / \mathrm{l}) \\
\text { Albumin }(\mathrm{g} / \mathrm{l})\end{array}$ & $\begin{array}{c}70.37 \pm 6.99 \\
40.9 \pm 6.03 \\
\end{array}$ & $\begin{array}{l}67.68 \pm 9.06 \\
38.01 \pm 8.36 \\
\end{array}$ & $\begin{array}{l}0.296 \\
0.214 \\
\end{array}$ \\
\hline $\begin{array}{c}\text { WBCs }(103 / \mu \mathrm{l}) \\
\text { Hb }(\mathrm{g} / \mathrm{dl}) \\
\text { Platelets }(103 / \mu)\end{array}$ & $\begin{array}{c}5.36 \pm 1.74 \\
9.45 \pm 1.24 \\
224.29 \pm 71.44\end{array}$ & $\begin{array}{c}6.75 \pm 2.21 \\
9.86 \pm 2.25 \\
254.16 \pm 65.33 \\
\end{array}$ & $\begin{array}{l}0.088 \\
0.474 \\
0.191\end{array}$ \\
\hline Mean ultrafiltration rate & $593.0 \pm 90.72$ & $621.14 \pm 108.3$ & 0.275 \\
\hline
\end{tabular}

Values are numbers $(\%)$ for categorical variables or mean \pm SD for continuous variables, (Median was added for data with wide SD) *. H CRP: high sensitivity C-reactive protein; HDL: high-density lipoprotein; LDL:

Low-density lipoprotein. WBCs: white blood cells. Hb: hemoglobin level. Statistically significant difference if $(\mathrm{p}<0.05)$

Table 4: Follow-up data of the patients comparing high flux and Low flux groups at baseline (0 time) and after 6 months.

\begin{tabular}{|c|c|c|c|}
\hline Item & Low flux & High flux & $\begin{array}{c}\text { P value between both } \\
\text { subgroups }\end{array}$ \\
\hline $\begin{array}{l}\text { S. albumin. } 0 \mathrm{~m} \\
\text { S. albumin. } 6 \mathrm{~m}\end{array}$ & $\begin{array}{c}40.9 \pm 6.03 \\
41.25 \pm 7.21\end{array}$ & $\begin{array}{l}38.01 \pm 8.36 \\
39.21 \pm 9.02\end{array}$ & $\begin{array}{c}P=0.214 \text { n.s. } \\
P=0.240\end{array}$ \\
\hline $\begin{array}{l}\text { Cholesterol. } 0 \mathrm{~m} \\
\text { Cholesterol. } 6 \mathrm{~m}\end{array}$ & $\begin{array}{l}158.81 \pm 33.99 \\
155.15 \pm 35.61\end{array}$ & $\begin{array}{c}164.08 \pm 49.05 \\
150.08 \pm 54.22^{* *}\end{array}$ & $\begin{aligned} P= & 0.694 \text { n.s } \\
& P<0.04\end{aligned}$ \\
\hline $\begin{array}{l}\text { TG. } 0 \mathrm{~m} \\
\text { TG. } 6 \mathrm{~m}\end{array}$ & $\begin{array}{l}171.67 \pm 74.61 \\
167.30 \pm 82.33\end{array}$ & $\begin{array}{c}181.54 \pm 88.68 \\
157.85 \pm 48.81^{* * *}\end{array}$ & $\begin{array}{c}P=0.463 \text { n.s } \\
P<0.03\end{array}$ \\
\hline $\begin{array}{l}\text { HDL. } 0 \mathrm{~m} \\
\text { HDL. } 6 \mathrm{~m}\end{array}$ & $\begin{array}{c}29.67 \pm 9.12 \\
30.89 \pm 10.04\end{array}$ & $\begin{array}{c}29.15 \pm 7.81 \\
36.08 \pm 15.02^{* *}\end{array}$ & $\begin{array}{l}P=0.863 \text { n.s } \\
P=0.148 \text { n.s }\end{array}$ \\
\hline $\begin{array}{l}\text { LDL. } 0 \mathrm{~m} \\
\text { LDL. } 6 \mathrm{~m}\end{array}$ & $\begin{array}{l}97.00 \pm 45.86 \\
95.05 \pm 43.49\end{array}$ & $\begin{array}{l}87.42 \pm 23.59 \\
85.16 \pm 48.11\end{array}$ & $\begin{array}{ll}P=0.078 & \text { n.s } \\
P=0.089 & \text { n.s }\end{array}$ \\
\hline $\begin{array}{l}\text { hCRP. } 0 \mathrm{~m} \\
\text { hCRP. } 6 \mathrm{~m}\end{array}$ & $\begin{array}{l}11.81 \pm 5.92 \\
12.98 \pm 2.40\end{array}$ & $\begin{array}{c}14.46 \pm 5.56 \\
7.61 \pm 2.35^{* *}\end{array}$ & $\begin{array}{c}P=0.154 \text { n.s } \\
P<0.001\end{array}$ \\
\hline $\begin{array}{l}\text { LDL. } 0 \mathrm{~m} \\
\text { LDL. } 6 \mathrm{~m}\end{array}$ & $\begin{array}{l}97.00 \pm 45.86 \\
95.05 \pm 43.49\end{array}$ & $\begin{array}{l}87.42 \pm 23.59 \\
85.16 \pm 48.11\end{array}$ & $\begin{array}{ll}P=0.078 & \text { n.s } \\
P=0.089 & \text { n.s }\end{array}$ \\
\hline
\end{tabular}

Values are mean \pm SD .h CRP, high sensitivity C-reactive protein; HDL, high-density lipoprotein;

LDL, low-density lipoprotein. Marked results point to statistically significant difference within the same group over time from baseline to $6 \mathrm{~ms}$ point.

$* \mathrm{p}<0.05 \quad * * \mathrm{p}<0.01 \quad * * * \mathrm{P}<0.001$ 


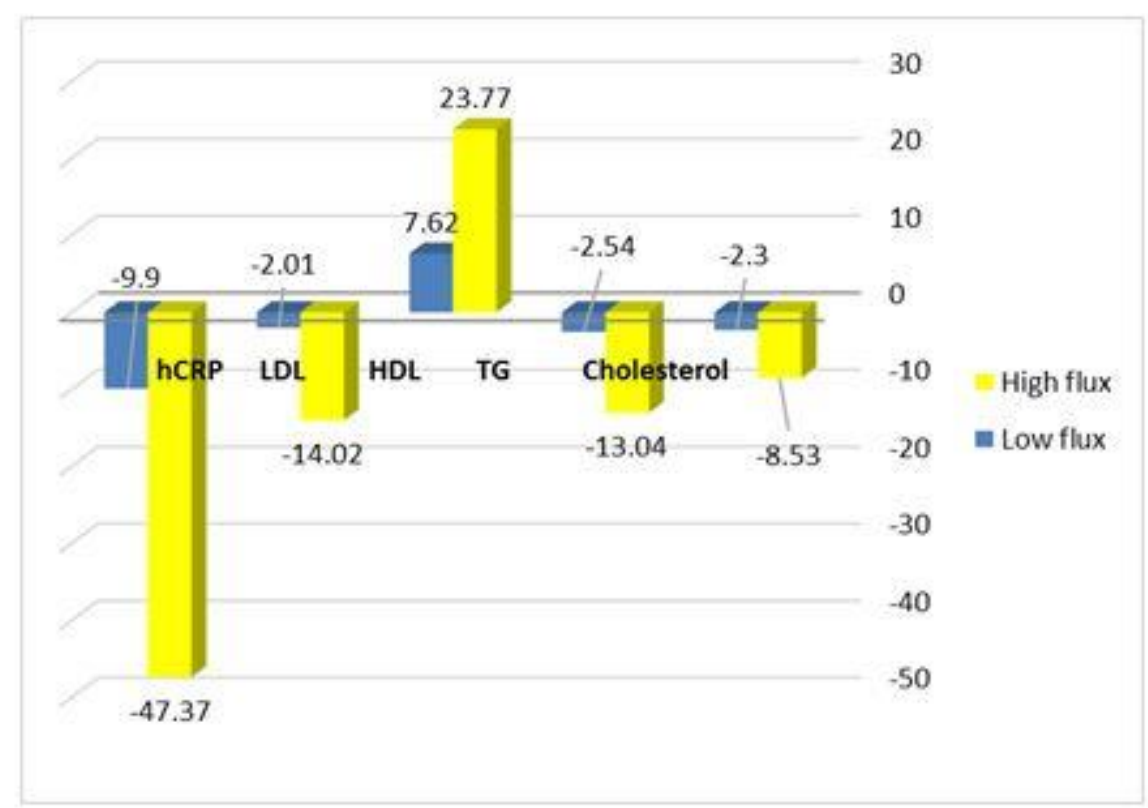

Graph (1) : Change in lipid parameters and h CRP of the two sub groups at the end of follow-up in terms of low-density lipoprotein (LDL)- Cholesterol, Total Cholesterol, highdensity lipoprotein (HDL)-Cholesterol, Triglyceride levels , and hs CRP

\section{Discussion}

While CVD-related mortality is relatively uncommon in young population, it accounts for most deaths in young CKD population [8]. The American Heart Association pediatric consensus guidelines for cardiovascular health stratified the uremic children into the highest tier of cardiovascular risk because of accelerated atherosclerosis [7], which originates early in childhood [9].

The pathogenesis of CVD in CKD is complex and involves multiple risk factors, of which dyslipidemia and inflammation are of major concern [10]. Uremic adult patients characteristically have hypertriglyceridemia, and reduced high-density lipoprotein (HDL) cholesterol [3]. In the field of pediatrics, uremic children receiving hemodialysis already have similar lipid abnormalities. However, a general limitation of defining dyslipidemia in childrenis lack of a comprehensive normative set of data that includes TG levels in young ( $<12$ years) children and significant variability in lipid values with respect to sex, age, and ethnicity [11].

In this study, we found that $81.3 \%$ of our patients had dyslipidemia. This is consistent with Khandelwal et al [9] who found dyslipidemia in $73.8 \%$ of their Indian patients. They used the same cut off points for dyslipidemia. However, Khandelwal studied patients with higher GFR values. The results of the present study are higher than what was reported by Saland [11] and Wilson [1] (only $45 \%$ and $44 \%$ of their American cohort). This may be attributed to less severe stages of CKD of their studied patients, or variation in the reference ranges for dyslipidemia used in these studies. The most common type of dyslipidemia in the present study was hypertriglyceridemia $(60 \%)$, similar to Khandelwal (73.8\%). However, it was much more prevalent than what was reported by Saland (only $32 \%$ ).
Reduced level of HDL was observed in $54 \%$ of our patients, which was higher than other studies $(41 \%$ in khandelwal and $21 \%$ in Saland et al). We reported elevated non HDL cholesterol in $20 \%$ of patients which is consistent with prevalence reported by khandelwal and Saland (27\% and $16 \%$ respectively). The dyslipidemia profile characteristic of pediatric CKD/ ESRD does not include an elevated LDL-C or total cholesterol as a prominent or consistent finding [5]. This is in accordance with our finding where elevated LDL and cholesterol levels was found in only $6 \%$, and $10 \%$ of patients respectively. Although, Khandelwal [9] found them in $30 \%$, and $37.5 \%$ of their cohort.

Although symptomatic coronary artery disease (CAD) is rarely noted in children with CKD, atherosclerosis is already evident in children with advanced kidney disease [2]. Subclinical atherosclerotic cardiovascular disease (ASCVD) during childhood has been discovered by sampling vasculature at the time of renal transplant [12]. In addition, young adult survivors of childhood ESRD experience an extremely high rate of premature mortality due to ASCVD $[13,14]$. Association of increased carotid intimal thickness (c IMT) (which is a validated predictor of cardiovascular risk in adults) with elevated triglycerides, total cholesterol, and LDL cholesterol has been found in children with various stages of CKD $[9,15,16,17]$. So the clinical significance of "atherogenic dyslipidemia" in this pediatric population lies in their potential risk of premature morbidity and mortality due to associated risk of CVD.

Recent data from clinical studies on adults imply that dialysis with high-flux synthetic membranes could attenuate hyperlipidemia. However, not all studies had reported beneficial effects of high-flux hemodialysis. Data are somewhat conflicting [3].

Comparing low-flux and high-flux dialyzers in the present study, has demonstrated a significant improvement 
in triglyceride and total cholesterol levels after 6 months of treatment with high flux dialyzers. This is in accordance with Goldberg et al [18], Wanner et al [19], Merello Godino et al [20] and Azak et al [3] who showed that highflux dialysis led to a significant improvement of triglyceride and total cholesterol concentrations after 1, 3, 6, 6 months of treatment respectively. Also, Josephson et al [21] reported improved hypertriglyceridemia in patients dialyzed with high-flux vs low-flux dialyzers. In addition, Dumler et al [22]. Found a significant decrease of triglycerides during 6 months of high-flux hemodialysis but with no significant effects on total and LDL cholesterol. Such possible beneficial effects could be related either to increased convective transport of large solutes with highflux dialysis or possibly by modifying associated oxidative stress. It is important to mention here that membrane material was the same in both subgroups and the difference was related only to the type of the dialyzer (high versus low). In contrast, House [23], and Tao Li [24] could not confirm these results and found non-significant decreases of triglyceride in the high-flux group as compared with the low-flux group. These studies were only for 3 months of follow up which may explain negative results while longer periods of treatment and follow-up may be essential in order to detect the positive contribution of high flux membranes in terms of dyslipidemia. In the present work, high flux dialyzers was associated with a significant increase in levels of protective HDL cholesterol when values after 6 months were compared with baseline one. However, there was no significant difference in HDL between both subgroups at 6 month. Azak et al found that high flux dialysis has beneficial effect on HDL cholesterol [3], while wanner et al didn't find similar effect [19]. As Drug free treatment for dyslipidemia seems to be of special importance in children, so demonstration of beneficial effect of high flux dialysis on total cholesterol, triglyceride, and to a certain extent on HDL for first time in uremic children is interesting.

Actually, we do not include any cardiovascular endpoints for any improvement of dyslipidemia. However, Morimoto et al [25] have reported that longer patient survival is characterized by being free of hypercholesterolemia and hypertriglyceridemia at the initiation of HD. In addition, Hinderliter et al [26] reported that traditional cardiovascular risk factors and higher maximum intimal media thickness (IMT) were independent predictors of cardiovascular events and death in patients with advanced pre-dialysis CKD.

The term Reverse epidemiology of hypercholesterolemia, where hypercholesterolemia appear to have protective features with a greater survival among dialysis patients in (ESRD) patients, is considered misleading by many authors as Levin et al [27]. They consider lower cholesterol as a marker of poor health and under nutrition and so is associated with adverse cardiovascular outcome in dialysis patients.

High sensitivity assay of $\mathrm{C}$ reactive protein (hs CRP) enables the identification of CRP at levels that were previously undetectable using routine assays [28]. So the American Heart Association recommends hs CRP as a more sensitive assay for the prediction of vascular disease, compared to traditional assays for CRP levels [29]. Renal insufficiency was independently associated with elevation of hs CRP, which may indicate an important pathway mediating the increased cardiovascular risk in CKD [30]. Hs CRP induces the expression of adhesion molecules such as intravascular adhesion molecule I ,vascular adhesion molecule I and E selectin which play a crucial role in the migration of monocytes and $\mathrm{T}$ leucocytes into vessel wall and thus in atherosclerosis [31]. Additionally, CRP may induce apoptosis in human coronary vascular smooth muscle cells, thus promoting atherogenesis [32]. So, elevated serum level of hs CRP does not only indicate an acute episode of inflammation in uremic patients but also signal a higher risk of cardiovascular complications.

This study provides evidence that hs CRP was significantly higher in uremic children than control. This is in accordance with Abraham et al, (Owen and Lowrie), and Shilpak who performed their studies on adult population $[30,33,34]$. The point of interest here is that hs CRP was reduced significantly by high flux dialysis, suggesting that high flux dialysis may be more protective against inflammatory process. Earlier adult studies didn't show similar results $[19,35]$, which may be attributed to shorter study duration or difference in the age of target population. Improvement of hs CRP may be attributed to removal of more soluble inflammatory mediators from the circulation by high flux dialysis. This may influence cellular activation levels in leukocytes. As malnutrition has important effect on the inflammatory milieu, it is important to mention here that baseline nutritional status of both subgroups was quite similar as percentage of patients with $\mathrm{z}$ score (BMI) below $3 \mathrm{SD}$ and the mean serum albumin were not significantly different at start of the study.

\section{Conclusion}

This study demonstrates a significant effect of high-flux hemodialysis on markers of uremic dyslipidemia and hs CRP. These results suggest that high-flux dialysis may have benefit in reducing the atherogenic risk and so CVS morbidity and mortality in uremic children.

Study Limitations There are some limitations in the current study such as the small available sample size, short duration of follow-up, monocentric experience of the study, lack of available data on caloric intake, lipid profile of the food. Further studies with longer durations are recommended on larger number of patients to confirm such findings

\section{Contributors'statement}

Dyslipidemia and inflammation are major risk factors for cardiovascular morbidities which are the main cause of mortality in uremic children. This study has demonstrated improvement of some markers of dyslipidemia and inflammation for the first time in uremic children by using high-flux hemodialysis.

\section{List of Abbreviations}

Body mass index (BMI)

Cardiovascular disease (CVD)

Chronic kidney disease (CKD)

End stage renal disease (ESRD) 
Increased carotid intimal thickness (cIMT)

Higher maximum intimal media thickness (IMT)

Hemodialysis (HD).

High sensitivity CRP (hs CRP)

Left ventricular hypertrophy (LVH)

\section{References}

1. Wilson C., Michael F., Christopher C. et al .Prevalence and Correlates of Multiple Cardiovascular Risk Factors in Children with Chronic Kidney Disease. Clinical Journal of the American Society of Nephrology: 2011, 2759- 2765.

2. Mitsnefes. Cardiovascular disease in children with chronic kidney disease. J Am Soc Nephrol; 2012, 23 (4): 578-85. doi: 10.1681/ASN.2011111115.

3. Azak A., Bülent H., Kürs, ad Öneç et al. Contribution of High Flux Membranes to the Therapy of UremiaAssociated Dyslipidemia. Therapeutic Apheresis and Dialysis, 2012, 16(6):595-599.

4. Akchurin M. Oleh and Kaskel Frederick. Update on Inflammation in Chronic Kidney Disease.

Blood Purif; 2015, 39:84-92 DOI: 10.1159/000368940

5. Greenbaum A. Larry, Bradley A. Warad, and Susan L. Furth. Current Advances in Chronic Kidney Disease in Children: Growth, Cardiovascular, and Neurocognitive Risk Factors Semin Nephrol. 2009, July; 29(4): 425-434.doi: 10.1016/j.semnephrol. 2009.03.017.

6. Daniels R., Frank R., and the Committee on Nutrition. Lipid Screening and Cardiovascular Health in Childhood, Pediatrics; 2008, 122:198-208.

7. Expert Panel on Integrated Guidelines for Cardiovascular Health and Risk Reduction in Children and Adolescents; National Heart, Lung, and Blood Institute. Expert panel on integrated guidelines for cardiovascular health and risk reduction in children and adolescents: summary report.

Pediatrics, 2011, 128 [Suppl 5]:S213-S256.

8. Mathews TJ, Minino AM, Osterman MJ. Annual summary of vital statistics:

Pediatrics,2008, 127: 146-157.

9. .Khandelwal P. \& Vijaya M. \& Smriti H. et al. Dyslipidemia, carotid intima-media thickness and endothelial dysfunction in children with chronic kidney disease.

Pediatr Nephrol, 2016, DOI 10.1007/s00467-016-3350-4. 10. Chauhan V1, Vaid M. Dyslipidemia in chronic kidney disease: managing a high-risk combination.

Postgrad Med.; 2009, 121(6):54-61.

doi: 10.3810/pgm.2009.11.2077.

11. Saland J, Christopher B, Mitsnefes M. Dyslipidemia in Children with Chronic Kidney Disease: A Report of the Chronic Kidney Disease in Children (CKiD) Study.

Kidney Int.; 2010, 78(11): 1154-1163.

doi:10.1038/ki.2010.311.

12. Nayir A, Bilge I, Kilicaslan I: Arterial changes in pediatric hemodialysis patients undergoing renal transplantation.

Nephrol Dial Transplant, 2001, 16:2041-2047.

13. U.S. Renal Data System. USRDS. Annual Data Report: Atlas of Chronic Kidney Disease and End-Stage Renal Disease in the United States. National Institutes of Health, National Institute of Diabetes and Digestive and
Kidney Diseases; Bethesda, MD: Bethesda, MD: USRDS Coordinating Center; 2008. http://www.usrds.org, 2008.

14. McDonald SP, Craig JC. Long-term survival of children with end-stage renal disease.

N Engl J Med; 2004, 350:2654-2662.

[PubMed: 15215481]

15. Brady TM, Schneider MF, Flynn JT, et al. Caro tid intima-media thickness in children with CKD: results from the CKiD study.

Clin J Am Soc Nephrol, 2012, 7:1930-1937.

16. Kotur-Stevuljevi ć J, Peco-Antic A, Spasic S,et al. Hyperlipidemia, oxidative stress, and intima media thickness in children with chronic kidney disease. Pediatr Nephrol 2013, 28:295-303.

17. Sozeri B, Mir S, Kara OD, et al.When does the cardiovascular disease appear in patients with chronic kidney disease? Pediatr Cardiol 2010, 31:821-828.

18. Goldberg IJ, Kaufman AM, Lavarias VA. High-flux dialysis membranes improve plasma lipoprotein profiles in patients with end-stage renal disease. Nephrol Dial Transplant; 1996, 1 [Suppl 2]: 104-107.

19. Wanner Ch., Udo B., Renate M. Effect of dialysis flux and membrane material on dyslipidaemia and inflammation in haemodialysis patients. Nephrol Dial Transplant 2004, 19: 2570-2575.

20. Merello Godino JI, Rentero R, Orlandini G, and Results from EuCliD (European Clinical Dialysis Database): impact of shifting treatment modality.

Int J Artif Organs; 2002, 25: 1049-1060.

21. Josephson MA, Fellner SK, Dasgupta A. Improved lipid profiles in patients undergoing high-flux hemodialysis. Am J kidney Dis; 1992, 20:361-6.

22. Dumler F, Stalla K, Mohini R. Clinical experience with short-time hemodialysis.

Am J Kidney Dis; 1992, 19: 49-56.

23. House AA, Wells GA, Donnelly JG. Randomized trial of high-flux vs low-flux haemodialysis: effects on homocysteine and lipids.

Nephrol Dial Transplant; 2000, 15: 1029-1034.

24. Tao Li Philip K, Yuk Lun Ch., $\uparrow$ Chi Bon L. Effect of Membrane Permeability on Inflammation and Arterial Stiffness: A Randomized Trial

Clin J Am Soc Nephrol.; 2010, 5(4): 652-658.

doi: $10.2215 /$ CJN.056208097.

25- Morimoto S, Nishioka H, Morita $\mathrm{T}$ et al. Characteristics of 20-year survivors undergoing maintenance hemodialysis.

Ther Apher Dial; 2010, 14:547-51.

26. Hinderliter A., Robin L. Padilla, and Rajiv Saran. Association of carotid intima-media thickness with cardiovascular risk factors and patient outcomes in advanced chronic kidney disease: the RRI-CKD study. Clin Nephrol, 2015, July 84(1): 10-20.

27. Levin NW1, Handelman GJ, Coresh J, Port FK. Reverse epidemiology: a confusing, confounding, and inaccurate term. Semin Dial. ; 2007, 20(6):586-92.

28. Ridker PM. High-sensitivity C-reactive protein potential adjunct for global risk assessment in the primary prevention of cardio-vascular disease.

Circulation; 2007, 103:1813-1818. 
29. American Heart Association Inflammation, Heart

Disease and Strok2, 2008.

http://www.americanheart.org/presenter.jhtml?identifier = 4648. Accessed December 2008.

30. Abraham Georgi, Varun Sundaram, Vivek Sundaram, et al. C-Reactive protein, a valuable predictive marker in chronic kidney disease Saudi J Kidney Dis Transpl.,2009, 20 (5): 811-815, Saudi Center for Organ Transplantation.

31. Corrado E1, Rizzo M, Coppola G. An update on the role of markers of inflammation in atherosclerosis.

J Atheroscler Thromb.; 2010, 17(1):1-11.

32. Blaschke F, Dennis B, Fen Y, et al. C - reactive protein Induces Apoptosis in Human Coronary Vascular Smooth Muscle Cells .Circulation. 2004, 110:579-587.

33. Owen WF, Lowrie EG. C-reactive protein as an outcome predictor for maintenance hemodialysis patients. Kidney Int 1988; 54:627-36.

34. Shilpak MG. Elevation of inflammatory and procoagulant biomarkers in elderly persons with renal insufficiency. Circulation; 2003, 107:32-7.

35. Akoglu H1, Dede F, Piskinpasa S.

Blood Purify. 2013, 35(4):258-64.

doi: 10.1159/000348453.

\section{Declaration}

\section{Ethical approval}

All procedures performed in the study were approved by Assiut University Ethical Committee and were in accordance with the 1964 Helsinki declaration and its later amendments. Informed consent: Informed written consent was obtained from guardians of all individual participants included in the study in accordance with Assiut University Ethical Committee guidelines.

\section{Conflict of interest}

The authors declare that they have no conflict of interest. No financial benefits have been received or will be received from any party related directly or indirectly to the subject of this article.

\section{Funding}

There is no funding source.

\section{Acknowledgements}

The authors wish to thank all patients who participated in this study and acknowledge the help of the laboratory and dialysis unit personals. 